\title{
Nueva metodología para evaluar las características enológicas de las unidades de un terroir. Vinificación de mistela.
}

\author{
Ernesto Franco $^{1}$, Miguel Lorente ${ }^{2}$ \\ ${ }^{1}$ Centro de Transferencia Agroalimentaria - Gobierno Aragón. Unidad de Enología. Avda. de Movera, \\ s/n, 50194, Zaragoza (España).efranco@aragon.es \\ ${ }^{2}$ Grupo de Estudios de Desarrollo Territorial, Universidad de Zaragoza (GEDETUZ). España. \\ mlorenteb@yhoo.es
}

\begin{abstract}
To To characterize terroir units that express the interaction between the vine and the geographical environment it is necessary to study the oenological parameters of each terroir. Nevertheless, this study presents many difficulties if the number of areas to study it is huge as it was the case of the zoning of DOP Campo de Borja. Grape analyses during harvest are expensive and give limited information. At the same time, fermentations in small-volume containers are difficult to control and introduce factors that are not directly related to the geographical environment. These small-scale fermentations can distort the link between the wine and the soil giving inaccurate results. In the project for the zoning of the DOP Campo de Borja has been used a new simple method which consists in vinification mistela. This new method consisted on producing a wine spirit directly from the grapes but without alcoholic fermentation. Therefore, with this new methodology of making mistelas, an wine produced without fermentation, was possible to obtain the contribution of the terroir of the DOP Campo de Borja to the composition of the grape and, consequently to the wines
\end{abstract}

\section{Introducción}

Esta presentación es el resultado del trabajo desarrollado por el Centro de Transferencia Agroalimentaria (CTA) en el "Proyecto de valorización de la D.O. Campo de Borja a través de la vinculación del vino con el territorio" encaminado a dotar del máximo prestigio comercial al vino de la D.O. Campo de Borja, vinculándolo a su territorio de origen como vector de desarrollo territorial, a partir del conocimiento de las aptitudes del medio geográfico que permita una gestión razonada del cultivo de la vid, de la elaboración de los vinos y de la comunicación en la comercialización.

Con el proyecto se pretende identificar y delimitar las áreas homogéneas, denominadas Unidades de Terroir, para gestionar la producción de vino bajo el concepto de calidad, autenticidad y originalidad. En definitiva, se pretende encontrar el vínculo entre los diferentes terroir que se delimiten en la D.O. y las características de los vinos de Garnacha cultivados en dichos terroir.

Ahora bien, en la calidad y características de los vinos, influyen numerosos factores, unos relacionados con la uva, como la vinífera, el terroir, madurez y sanidad y otros relacionados con la vinificación y conservación del vino, como levadura, maceración, prácticas enológicas, crianzas, etc. Sin lugar a dudas estas últimas modifican sustancialmente las características del vino y pueden disminuir el peso de la vinífera y del terroir.
En base a lo anterior, y teniendo en cuenta que el análisis de uvas en la fecha de la vendimia es costoso y la información obtenida limitada y que la fermentación en recipientes de poco volumen es difícil de controlar e introduce factores que no están directamente relacionados con el medio geográfico y pueden desvirtuar el vínculo entre el vino y el territorio, por lo que los resultados a veces resultan erróneos, se opta por una vinificación sin fermentación alcohólica, mistela, un tipo de vino de licor, mosto o uva estrujada al que se adiciona alcohol de origen vínico y definida en el anexo IV del Real Decreto $1127 / 2003$ y posteriores reglamentos CE. Se parte de la experiencia previa de vinificación de mistelas, línea de recuperación de vinos dulces tradicionales que desarrolla el CTA desde 1995.

En las mistelas al no producirse fermentación, tanto los aromas como polifenoles proceden solo y exclusivamente de la uva, y si bien el alcohol no es inodoro su papel es evitar la fermentación y ejercer acciones de disolvente y esterificación, favoreciendo la expresión aromática de la variedad por procesos de hidrólisis tanto química como enzimática.

\section{Objetivo}

Es postular la vinificación, de mistela tinta con uvas de Garnacha Tinta, como una herramienta para establecer el vínculo entre las características analíticas y sensoriales del vino y el terroir. Relacionando las características de 
las mistelas con los vinos tintos obtenidos de las mismas parcelas.

\section{Material y métodos}

Las vinificaciones de mistelas tintas y vinos tintos se han realizado durante los años 2012, 2013 y 2014, en el Proyecto se ha trabajado con 79 parcelas, si bien en este estudio solo se muestran las 23 parcelas que han sido objeto de vinificación en los tres años de trabajo. Un total de 23 parcelas de diferentes terroir de la D.O.P. Campo de Borja, se han vinificado cada año como mistela y además en seis de ellas se han vinificado vinos tintos.

\subsection{Viníficación de mistela tinta}

3.1.1 La uva de Garnacha Tinta de cada terroir se vendimia en el entorno de la madurez que marca la Bodega de la D.O.P. Campo de Borja receptora de la uva de esa parcela.

3.1.2 Se vendimia en cajas de $25 \mathrm{Kg}, 50 \mathrm{Kg}$ de uva de cada parcela.

3.1.3 En campo se controla la producción mediante el dato de $\mathrm{Kg}$ /cepa, trasladándola a la uva a la Bodega Cava de Almonacid de la Sierra del CTA.

3.1.4 La uva se despalilla y estruja y se encuba en depósitos de acero inoxidable de 251 de capacidad, dosificando $40 \mathrm{mg} / \mathrm{kg}$ de anhídrido sulfuroso en forma de solución acuosa del 15\%.

3.1.5 Transcurrido 30 minutos de la operación anterior, se procede a la adición de $140 \mathrm{cc} / \mathrm{Kg}$ de uva de alcohol de $96 \%$, homogeneizando toda la masa.

3.1.6 Diariamente, y de forma manual, se homogeneiza toda la uva del depósito. La temperatura se mantiene constante a $12^{\circ} \mathrm{C}$.

3.1.7 Después de siete días de maceración se prensa, y la mistela obtenida se corrige hasta $15 \%$ de alcohol si es necesario.

3.1.8 Transcurridos 15 días de la prensa se procede al trasiego para retirar lias, dosificando $30 \mathrm{mg} / 1$ de anhídrido sulfuroso en forma de solución acuosa al 15\%

3.1.9 Transcurridos 30 días de la operación anterior se procede a un segundo trasiego para retirar lias, si fuera necesario se dosificará anhídrido sulfuroso, para mantener el nivel de sulfuroso libre en $20 \mathrm{mg} / \mathrm{l}$.

\subsection{Viníficación de vino tinto}

De seis parcelas en las que se han vinificado mistelas, se realizan vinificaciones semi industriales de vino tinto, siguiendo los siguientes pasos:

3.2.1 La uva de Garnacha Tinta de cada terroir se vendimia a la vez que su correspondiente vendimia de mistela como se describe en 3.11.

3.2.2 Se vendimia en cajas de $25 \mathrm{Kg}, 1200 \mathrm{Kg}$ de uva de cada parcela.
3.2.3 En campo se controla la producción mediante el dato de $\mathrm{Kg} / \mathrm{cepa}$, trasladándola a la uva a la Bodega Piloto de Ainzón del CTA.

3.2.4 La uva se despalilla y estruja, se encuba en depósitos de acero inoxidable de $15 \mathrm{hl}$ de capacidad, dosificando $40 \mathrm{mg} / \mathrm{kg}$ de anhídrido sulfuroso en forma de solución acuosa al $15 \%$

3.2.5 Se inocula levadura seca activa, de acción mecánica, a razón de $20 \mathrm{~g} / \mathrm{Kg}$ de uva y activadores de fermentación.

3.2.6 Diariamente se realizan dos remontados, la maceración se prolonga durante 10 días después de iniciada la fermentación alcohólica.

3.2.7 Transcurrido el periodo anterior la uva se prensa, favoreciendo posteriormente la degradación maloláctica.

3.2.8 Concluida de degradación maloláctica o transcurridos 30 días desde el final de la fermentación alcohólica, se procede al trasiego para retirar lias, dosificando $50 \mathrm{mg} / \mathrm{l}$ de anhídrido sulfuroso en forma de solución acuosa al $15 \%$

3.2.9 Transcurridos 30 días de la operación anterior se procede al segundo trasiego para retirar lias, si fuera necesario se dosificará anhídrido sulfuroso, para mantener el nivel de sulfuroso libre en $25 \mathrm{mg} / \mathrm{l}$.

\subsection{Métodos analíticos}

En las mistelas se han analizado los siguientes parámetros, densidad relativa, grado alcohólico, azúcares reductores, acidez total, $\mathrm{pH}$, sulfuroso libre y total, ácido málico, potasio, intensidad de color e IPT. En los vinos se han analizado, densidad relativa, grado alcohólico, azúcares reductores, acidez volátil, acidez total, $\mathrm{pH}$, sulfuroso libre y total, ácido málico, potasio, intensidad de color e IPT. En ambos casos se han empleado métodos habituales y oficiales de análisis.

\subsection{Estadística}

En el estudio estadístico se utilizan técnicas de análisis de regresión, análisis exploratorio de Tukey (estadística descriptiva) y análisis multivariante (análisis de componentes principales ACP). Dichas técnicas se aplicaron utilizando el programa informático SPSS

\subsection{Metodología sensorial}

El análisis sensorial descriptivo, se realiza conjuntamente entre jurados del CTA, LAAE (Universidad de Zaragoza) y Comité de cata de la D.O.P. Campo de Borja. El panel lo componen 11 jueces experimentados y para la descripción de las mistelas y vinos se utilizan las fichas de las Figuras 1 y 2 . Se valora el color, aroma y gusto, el jurado debe puntuar entre 1 y 3 puntos cada uno de los descriptores. La puntuación de cada descriptor se obtiene ponderando su valor como se muestra en las fichas, la puntuación final es la suma del valor parcial de cada descriptor. En las fichas se consideran descriptores negativos en cuyo caso la ponderación es negativa y por tanto estos restan de la 
puntuación total. Este modelo permite trabajar con datos de organolépticos parciales, aroma, gusto y/o los totales, posibilitando a su vez relacionarlos con parámetros de viñedo y con la composición analítica de mistelas y vinos.

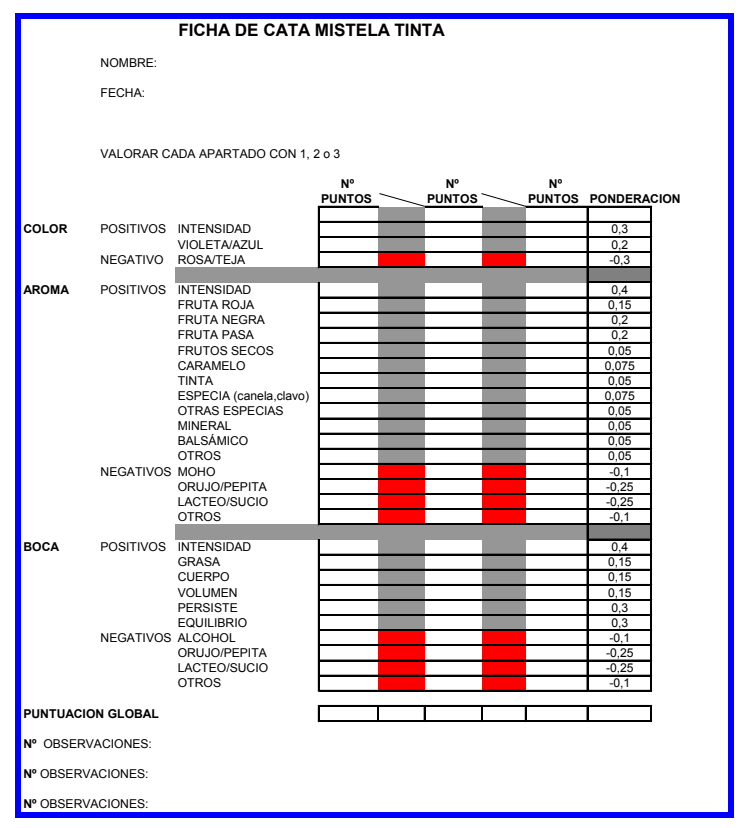

Figura 1. Ficha descriptiva de mistelas tintas.

Además, se pedía a los catadores una valoración global de cada mistela o vino que serviría para relacionarla con la puntuación total de los descriptores con el fin de conocer la bondad de la evaluación. En el análisis de regresión entre ambos parámetros se produce una correlación alta entre ambos valores $(\mathrm{r}=0,91$ en mistelas y 0,97 en vinos), lo que nos indica que el trabajo del jurado fue muy coherente en sus apreciaciones.

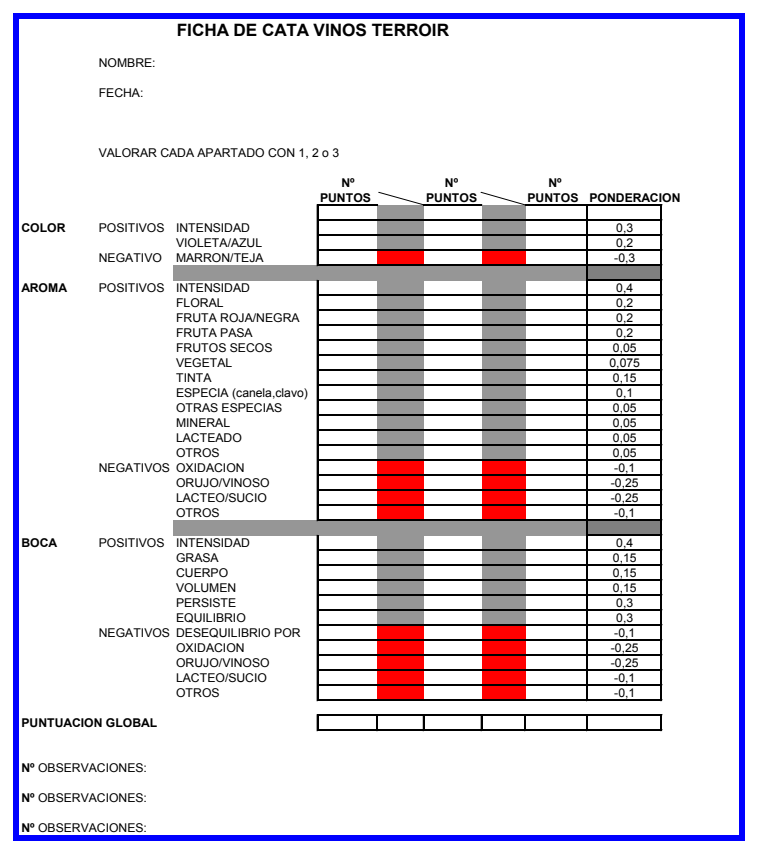

Figura 2. Ficha descriptiva de vinos tintos.

\section{Resultados}

\subsection{Agronómicos}

Se realiza un seguimiento de maduración de cada una de las parcelas conjuntamente con la Bodega que recepciona la uva, se observa si hay anomalías en la misma y se controla la producción. En la tabla 1 se muestran datos estadísticos de la producción en los tres años de ensayo.

Tabla 1. Producción por años de las 23 parcelas objeto de vinificación.

\begin{tabular}{|c|c|c|c|c|}
\hline & $2^{201}$ & $13^{20}$ & 14 & 20 \\
\hline ia & $2^{1,8}$ & $5^{1,4}$ & 9 & 3,4 \\
\hline${ }_{\text {imo }}^{\text {Máx }}$ & $6^{4,7}$ & $3^{3,1}$ & 5 & 6,2 \\
\hline imo & $4^{0,7}$ & 0,4 & 8 & 0,9 \\
\hline$v^{\text {Des }}$ & $8^{0,9}$ & $4^{0,7}$ & 5 & 1,5 \\
\hline
\end{tabular}

El rendimiento medio de las parcelas en 2012 y 2013 es similar, mientras que en 2014 fue muy superior a la de los dos años anteriores, observando en los tres años una desviación muy elevada. Es de destacar que en 2013 se produjo un corrimiento físiológico importante de la variedad Garnacha, que afecto con gran intensidad en todo Aragón.

\subsection{Analíticos y sensoriales de las mistelas.}

Teniendo en cuenta que la acidez y el pH se modifican sustancialmente por la adición de alcohol, ambos parámetros se descartan como elementos de valoración analítica de las mistelas. El análisis de componentes principales (ACP) muestra que el primer factor recoge la aportación principal de la intensidad de color y en menor medida de IPT y el segundo factor del azúcar, como se muestra en la figura 3. De acuerdo con lo anterior, los parámetros analíticos estudiados son azúcar, intensidad de color e IPT en mistelas. Los resultados analíticos de las mistelas de los años 2013 y 2014 son muy similares, en ambos años se observa precipitaciones similares, tabla 2, pero con diferente producción, la de 2014 es más de dos veces superior a la de 2013. En 2012 las mistelas son más concentradas en los tres parámetros, estas diferencias se plasman en particular en la intensidad de color e IPT, tabla 3, y que se podrían relacionar con una menor precipitación en 2012. 
Tabla 2. Datos climáticos básicos en los tres años de estudio.

\begin{tabular}{|l|c|c|c|}
\hline Observatorio Borja & $\mathbf{2 0 1 2}$ & $\mathbf{2 0 1 3}$ & $\mathbf{2 0 1 4}$ \\
\hline Pp anual (mm) & 336,6 & 504,9 & 543,1 \\
\hline Pp Jun-Jul (mm) & 38,8 & 57,3 & 42,2 \\
\hline${ }^{\mathbf{o}} \mathbf{C}$ T. media anual & 14,7 & 13,9 & 14,8 \\
\hline${ }^{\circ} \mathbf{C}$ T. media Junio & 22,8 & 18,4 & 20,8 \\
\hline
\end{tabular}

En el caso del análisis sensorial los años 2012 y 2014 son más parecidos, mientras que las mistelas del 2013 son las peor valoradas, tabla 3. La relación entre el aroma y gusto se muestra en la figura 4, la distribución de las valoraciones es uniforme y homogénea, es decir los criterios de cata utilizados en los tres años son idénticos, aspecto básico para una posterior clasificación de las mistelas. Dado que la puntuación del aroma y del gusto es la suma de los diferentes descriptores, a su vez estos también se convierten en una herramienta para relacionar la cualidad del aroma con el terroir.

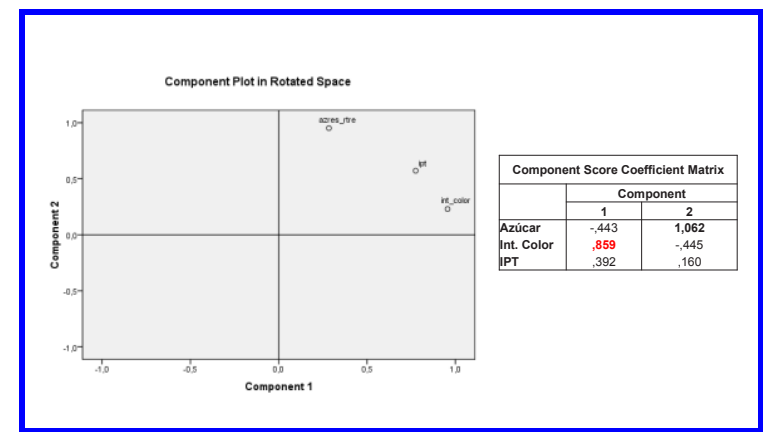

Figura 3. ACP de los parámetros analíticos de las mistelas. Años 2012, 2013 y 2014.

El análisis de componentes principales (ACP) de los descriptores del aroma, que son los relacionados en la figura 1, muestra que los que mayor capacidad de discriminación tienen son fruta roja, negra y pasa, junto con balsámico y tinta, figura 5. En la componente primera tienen más peso fruta negra, pasa y tinta, mientras en la componente segunda pesan más la fruta roja y balsámico.

Tabla 3. Valores analíticos y sensoriales de las mistelas por años

\begin{tabular}{|c|c|c|c|c|c|c|c|}
\hline Año & Valores & Azúcar & Color & IPT & Aroma & Gusto & Total \\
\hline & Media & 274,00 & 9,92 & 71,67 & 1,89 & 3,04 & 6,23 \\
\hline $\mathbf{2 0 1 2}$ & Máximo & 311,00 & 20,77 & 105,00 & 2,71 & 3,91 & 8,06 \\
\hline & Mínimo & 205,00 & 2,16 & 26,90 & 0,84 & 1,88 & 3,38 \\
\hline & Desv & 20,68 & 4,22 & 19,44 & 0,52 & 0,61 & 1,33 \\
\hline & Media & 249,56 & 6,79 & 38,70 & 1,60 & 2,75 & 5,43 \\
\hline $\mathbf{2 0 1 3}$ & Máximo & 282,50 & 17,30 & 57,30 & 2,41 & 3,62 & 7,45 \\
\hline & Mínimo & 207,00 & 2,05 & 21,80 & 0,41 & 1,78 & 1,78 \\
\hline & Desv & 19,14 & 3,55 & 9,44 & 0,56 & 0,50 & 1,54 \\
\hline
\end{tabular}

\begin{tabular}{|l|c|c|c|c|c|c|c|} 
& Media & 246,57 & 7,93 & 47,88 & 1,89 & 3,05 & 6,06 \\
\hline $\mathbf{2 0 1 4}$ & Máximo & 320,00 & 15,71 & 79,80 & 2,56 & 4,04 & 7,94 \\
\hline & Mínimo & 189,00 & 0,30 & 11,30 & 1,13 & 2,11 & 3,88 \\
\hline & Desv & 26,96 & 3,84 & 16,29 & 0,41 & 0,51 & 1,06 \\
\hline
\end{tabular}

Azúcar reductor en g/l; I. Color Abs 420+520+620 nm; IPT Abs 280nm.

Un aspecto importante es la producción del viñedo y cómo puede afectar a las características de las mistelas y los vinos, cabe preguntarse ¿Qué relación se puede observar entre los parámetros analíticos, sensoriales y la producción del viñedo?

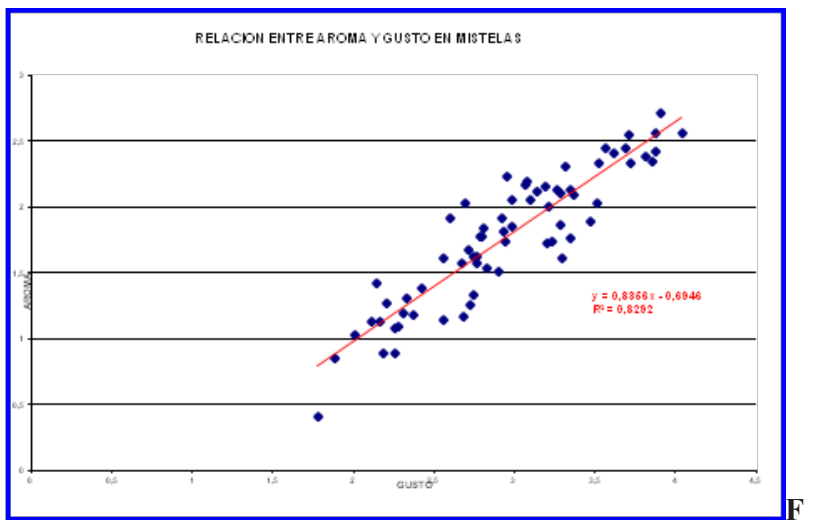

igura 4. Relación entre el aroma y el gusto de las valoraciones de los diferentes descriptores del aroma y del gusto de las mistelas de los años 2012,2013 y 2014.

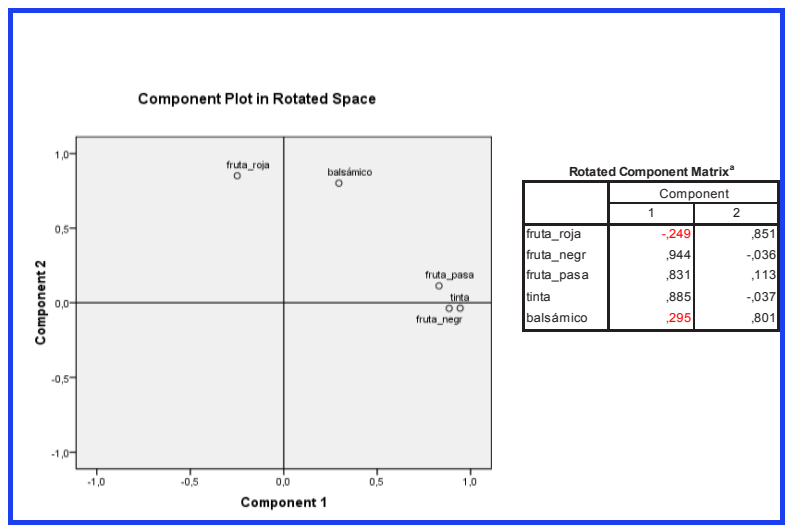

Figura 5. ACP de los descriptores del aroma de las mistelas. Años 2012, 2013 y 2014.

En la tabla 4; se muestra la relación entre los diferentes parámetros analítico, el análisis sensorial y la producción del viñedo, la tendencia es que a mayor producción los valores analíticos son menores y no observando relación entre el aroma y el gusto con la producción. Estas observaciones son muy importantes, dado que no ligan la analítica y los parámetros sensoriales de las mistelas con la producción, por tanto, las diferencias observadas entre las mistelas mayoritariamente se deben a otros factores diferentes a la producción, como los relacionados con el terroir.

También hay que destacar que los parámetros analíticos tampoco están relacionados con los sensoriales y solo el color tiene cierta relación con el sensorial total, 
dado que este incluye la valoración sensorial del color de la mistela. La independencia entre los parámetros analíticos y los sensoriales, indica que el factor terroir tiene un peso importante en un entorno de madurez similar.

Se observa una cierta relación entre IPT y la intensidad de color. La relación entre los parámetros sensoriales es muy buena e indica que cualquiera de ellos puede ser usado para valorar la calidad global de las mistelas.

\subsection{Analíticos y sensoriales de los vinos.}

En el caso de vinos tintos se tienen en cuenta todos los parámetros relacionados directamente con las características del vino, grado de alcohol, acidez total, $\mathrm{pH}$, intensidad de color e IPT. El análisis de componente principales, $\mathrm{ACP}$, de los parámetros analíticos, figura $\mathbf{6}$, nos indica que la componente primera se debe a la intensidad de color, $\mathrm{pH}$ y acidez total y la segunda al grado e IPT, estos parámetros analíticos son muy similares a los que también se relacionan con las características de las mistelas.

Tabla 4. Coeficientes de correlación (r) de parámetros analíticos, sensoriales y producción de las mistelas

\begin{tabular}{|l|c|c|c|c|c|c|}
\hline & Azúcar & Color & IPT & Aroma & Gusto & Total \\
\hline Producción & $-0,33$ & -0.02 & $-0,13$ & 0,00 & 0,00 & 0,00 \\
\hline Azúcar & & 0,51 & 0,73 & 0,28 & 0,30 & 0,35 \\
\hline I. Color & & & 0,82 & 0,54 & 0,62 & 0,70 \\
\hline IPT & & & & 0,44 & 0,48 & 0,56 \\
\hline Aroma & & & & & 0,91 & 0,93 \\
\hline Gusto & & & & & & 0,94 \\
\hline
\end{tabular}

En los vinos no se observan diferencias en cuanto al grado, como si se observaba en el azúcar de las mistelas y si en la intensidad de color e IPT, tabla $\mathbf{5}$, en el análisis sensorial los años 2013 y 2014 son más parecidos y los vinos del 2012 son los mejor valorados.

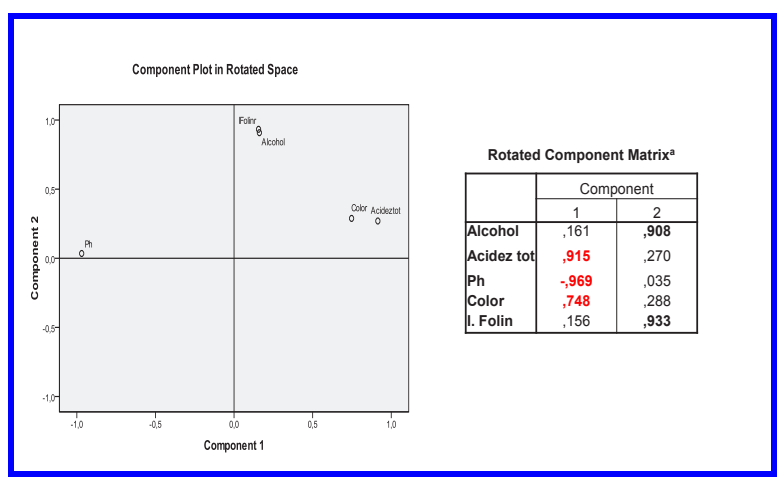

Figura 6. ACP de los parámetros analíticos de los vinos. Años 2012, 2013 y 2014

Tabla 5. Valores analíticos y sensoriales de los vinos años.

\begin{tabular}{|l|l|l|l|l|l|l|l|}
\hline Año & Valores & Grado & Color & IPT & Aroma & Gusto & Total \\
\hline
\end{tabular}

\begin{tabular}{|l|c|c|c|c|c|c|c|} 
& Media & 16,47 & 13,54 & 77,43 & 2,25 & 3,17 & 6,79 \\
\hline $\mathbf{2 0 1 2}$ & Máximo & 0,85 & 2,25 & 7,12 & 0,28 & 0,40 & 0,67 \\
\hline & Mínimo & 17,30 & 17,62 & 86,80 & 2,55 & 3,69 & 7,65 \\
\hline & Desv & 15,50 & 11,07 & 66,30 & 1,81 & 2,71 & 5,94 \\
\hline & Media & 16,30 & 15,40 & 74,97 & 2,02 & 2,73 & 5,93 \\
\hline $\mathbf{2 0 1 3}$ & Máximo & 0,89 & 3,53 & 8,81 & 0,54 & 0,86 & 1,58 \\
\hline & Mínimo & 17,50 & 18,24 & 85,50 & 2,60 & 3,82 & 7,83 \\
\hline & Desv & 14,95 & 10,60 & 63,00 & 1,38 & 1,56 & 3,66 \\
\hline & Media & 16,66 & 12,52 & 67,72 & 1,72 & 2,93 & 5,91 \\
\hline $\mathbf{2 0 1 4}$ & Máximo & 1,53 & 4,47 & 15,58 & 0,35 & 0,47 & 1,03 \\
\hline & Mínimo & 18,30 & 18,50 & 91,68 & 2,12 & 3,52 & 6,87 \\
\hline & Desv & 14,35 & 6,72 & 48,36 & 1,19 & 2,42 & 4,37 \\
\hline
\end{tabular}

La relación entre los parámetros analíticos, los sensoriales y entre todos ellos se muestra en la tabla $\mathbf{6}$, solo se observa una buena relación del IPT con la intensidad de color y el grado, y también con el aroma. Como también ocurre en las mistelas si hay alta relación del aroma y del gusto con la valoración total del vino.

Tabla 6. Coeficientes de correlación (r) de parámetros analíticos y sensoriales de los vinos

\begin{tabular}{|l|c|c|c|c|c|}
\hline & I. Color & IPT & Aroma & Gusto & Total \\
\hline Grado & 0,52 & 0,81 & 0,49 & 0,47 & 0,53 \\
\hline I. Color & & 0,79 & 0,62 & 0,46 & 0,61 \\
\hline IPT & & & 0,85 & 0,65 & 0,77 \\
\hline Aroma & & & & 0,79 & 0,93 \\
\hline Gusto & & & & & 0,95 \\
\hline
\end{tabular}

\subsection{Relación entre los parámetros analíticos y sensoriales de vinos y mistelas}

El efecto añada es mucho menor en los vinos que en las mistelas, como se observa al comparar los datos de las tablas 3 y 5. En el análisis sensorial tanto las mistelas como los vinos de 2012 fueron los mejor valorados, los vinos de los años 2013 y 2014 son más parecidos, a diferencia de las mistelas que eran 2012 y 2014.

La relación de los parámetros analíticos y sensoriales entre los vinos y las mistelas se muestra en la tabla 7, se observa mejor relación entre los parámetros analíticos que entre los sensoriales, siendo la relación del IPT y de azúcar/grado las que muestran la mayor correlación. No obstante, la correlación entre mistelas y vinos, para los seis parámetros estudiados, es suficientemente buena para poder relacionar las características de las mistelas con la de los vinos tintos obtenidos de las mismas parcelas.

Tabla 7. Coeficientes de correlación (r) de parámetros analíticos y sensoriales entre mistelas y vinos

\begin{tabular}{|c|c|c|c|c|c|c|}
\hline VINO & Grado & Color & IPT & Aroma & Gusto & Total \\
\hline MISTELA & & & & & & \\
\hline
\end{tabular}




\begin{tabular}{|l|l|l|l|l|l|l|}
\hline Azúcar & 0,76 & & & & & \\
\hline Color & & 0,74 & & & & \\
\hline IPT & & & 0,76 & & & \\
\hline Aroma & & & & 0,71 & & \\
\hline Gusto & & & & & 0,73 & \\
\hline Total & & & & & & 0,71 \\
\hline
\end{tabular}

\section{Conclusiones}

La acidez total y el $\mathrm{pH}$ no se pueden usar como parámetros de valoración analítica de las mistelas, mientras que azúcar, intensidad de color e IPT son los parámetros que discriminan. En el caso de los vinos son también estos parámetros los que diferencian.

La producción del viñedo no se relaciona con los parámetros analíticos y sensoriales de las mistelas. En 2012 las mistelas y los vinos son más concentrados en los tres parámetros analíticos y los mejor valorados sensorialmente, estas diferencias podrían relacionar con una menor precipitación en 2012. No se liga la analítica y los parámetros sensoriales de las mistelas con la producción, por tanto, las diferencias observadas entre las mistelas mayoritariamente se deben a otros factores diferentes a la producción, como los relacionados con el terroir.

De los descriptores del aroma de las mistelas la mayor capacidad de discriminación se debe a los descriptores fruta negra, pasa y tinta en la componente primera y fruta roja y balsámico en la segunda componente.

La independencia entre los parámetros analíticos y los sensoriales de las mistelas, muestran que el factor terroir tiene un peso importante en un entorno de madurez similar.

Se confirma el postulado que la vinificación, de mistela tinta con uvas de Garnacha Tinta, como herramienta para establecer el vínculo entre las características analíticas y sensoriales del vino y el terroir es adecuada, dado. La correlación de los seis parámetros estudiados, es suficientemente buena para poder relacionar las características de las mistelas con la de los vinos tintos obtenidos de las mismas parcelas.

\section{Referencias}

Real Decreto 1127/2003. BOE, núm. 228, de 23 de septiembre de 2003, pág 34823-34828 